JAWAD F., KARPENKO H., ZAGORODNYUK A.

\title{
ALGEBRAS GENERATED BY SPECIAL SYMMETRIC POLYNOMIALS ON $\ell_{1}$
}

Let $X$ be a weighted direct sum of infinity many copies of complex spaces $\ell_{1} \oplus \ell_{1}$. We consider an algebra consisting of polynomials on $X$ which are supersymmetric on each term $\ell_{1} \oplus \ell_{1}$. Point evaluation functionals on such algebra gives us a relation of equivalence ' $\sim$ ' on $X$. We investigate the quotient set $X / \sim$ and show that under some conditions, it has a real topological algebra structure.

Key words and phrases: symmetric and supersymmetric polynomials on Banach spaces, algebras of analytic functions on Banach spaces, spectra algebras of analytic functions.

\footnotetext{
Vasyl Stefanyk Precarpathian National University, 57 Shevchenka str., 76018, Ivano-Frankivsk, Ukraine

E-mail: farah.jawad@yahoo.com(Jawad F.), ganna.karpenko@gmail.com (Karpenko H.), azagorodnegmail.com (Zagorodnyuk A.)
}

\section{INTRODUCTION AND PRELIMINARIES}

Let $X$ be a complex Banach space and $\left(P_{\alpha}\right)$ a family of continuous complex valued polynomials on $X$. Often, it is interesting to consider algebras of analytic functions on $X$, generated by the family of polynomials (see e. g. $[6,12,16])$. If the family $\left(P_{\alpha}\right)$ does not separate points of $X$, then the same is true for any function, generated by $\left(P_{\alpha}\right)$. So, we have a natural relation of equivalence on $X: z \sim w$ if and only if $P_{\alpha}(z)=P_{\alpha}(w)$ for every $\alpha$. If $X$ is finite-dimensional, then from the Algebraic Geometry is well known that the quotient set $X / \sim$ is dens in an algebraic variety. The same is true for infinite-dimensional case, if the family $\left(P_{\alpha}\right)$ is finite [2]. But in the general case, the situation may be more complicated.

Let $S$ be the group of all permutations on the set of natural numbers $\mathbb{N}$. A polynomial $P: \ell_{1} \rightarrow \mathbb{C}$ is said to be symmetric if $P(\sigma(x))=P(x)$ for every $X \in \ell_{1}$ and $\sigma \in S$. It is known [15] that polynomials

$$
F_{k}(X)=\sum_{n=1}^{\infty} x_{n}^{k}, k=1,2, \ldots,
$$

form an algebraic basis in the algebra of all continuous symmetric polynomials $\mathcal{P}_{s}\left(\ell_{1}\right)$. In other words, $\left\{F_{k}\right\}_{k=1}^{\infty}$ are algebraically independent and $\mathcal{P}_{s}\left(\ell_{1}\right)$ is the minimal unital algebra containing $\left\{F_{k}\right\}_{k=1}^{\infty}$. In [1] it was shown that two vectors with finite supports $x, y \in \ell_{1}$ are equivalent in the means $F_{k}(x)=F_{k}(y)$ for every $k$, if and only if $x=\sigma(y)$ for some $\sigma \in S$. Some algebraic operations on $\ell_{1} / \sim$ which form a semi-ring structure [4] were considered in [5,7]. Composition operators, associated with these operations, on analytic functions were investigated in [8]. Algebras of analytic functions generated by symmetric polynomials on $\ell_{p}$ were investigated in $[1,3,5-7,13,14]$.

\section{$\mathrm{y} \Delta \mathrm{K} 517.98$}

2010 Mathematics Subject Classification: 46J15, 46J20.

The work was partially supported by Ministry of Education and Science of Ukraine Grant 0119 U100063 
Let $X=\ell_{1} \oplus \ell_{1}$. We represent each element $z$ of $X$ by $z=(y \mid x), x, y \in \ell_{1}$. Let us consider polynomials $T_{m}: X \rightarrow \mathbb{C}$,

$$
T_{m}(z)=F_{m}(x)-F_{m}(y)=\sum_{k=1}^{\infty}\left(x_{k}^{m}-y_{k}^{m}\right)
$$

Polynomials $T_{m}, m \in \mathbb{N}$ are algebraically independent and form an algebraic basis on the algebra of supersymmetric polynomials on X. In [11] the algebra of supersymmetric polynomials was investigated and a commutative ring structure on the corresponding quotient set $X / \sim$ was described.

For a given complex Banach space $E$ with an unconditional basis $\left\{e_{n}\right\}_{n=0}^{\infty}$ we denote by $\ell_{1}^{(E)}$ a Banach space defined by the following way. If $x \in \ell_{1}^{(E)}$, then

$$
x=\left(x^{(0)}, x^{(1)}, \ldots, x^{(n)}, \ldots\right),
$$

where each $x^{(n)}=\left(x_{1}^{(n)}, \ldots, x_{k}^{(n)}, \ldots\right) \in \ell_{1}$ and

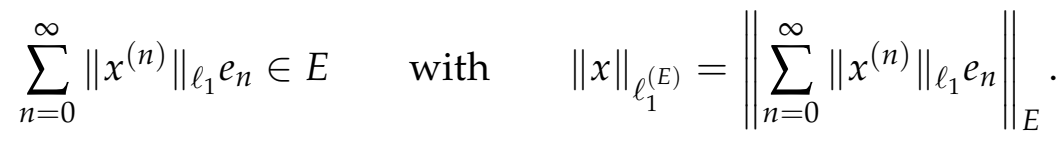

A polynomial $P$ on $\ell_{1}^{(E)}$ is separately symmetric [10] if for every sequence of permutations on $\mathbb{N}, \sigma=\left(\sigma_{0}, \sigma_{1}, \ldots, \sigma_{n}, \ldots\right), \sigma_{n} \in S$ we have $P(\sigma(x))=P\left(\sigma_{0}\left(x^{(0)}\right), \ldots, \sigma_{n}\left(x^{(n)}\right), \ldots\right)=P(x)$ for all $x \in \ell_{1}^{(E)}$. Polynomials

$$
F_{m}^{(j)}(x)=\sum_{k=1}^{\infty}\left(x_{k}^{(j)}\right)^{m}, \quad j \in \mathbb{Z}_{+}, \quad m \in \mathbb{N}
$$

are separately symmetric and algebraically independent.

In this paper we consider a complex Banach space $X$ which is a weighted direct sum of infinity copies of $\ell_{1} \oplus \ell_{1}$ and polynomials which are supersymmetric on each term of this sum. We show that under some assumptions, $X / \sim$ is a real locally convex algebra which contains a normed subalgebra. This is an extension of results on supersymmetric polynomials, obtained in [11]. For details about analytic mappings on Banach spaces we refer the reader to [9].

\section{THE RING $\mathcal{M}^{\omega}$}

Let $\omega$ be a positive number, $0<\omega \leq 1$. We denote by $\ell_{1, \infty}^{\omega}$ a "weighted" version of the space $\ell_{1}^{E}$. Namely, if $x \in \ell_{1, \infty}^{\omega}$, then

$$
x=\left(x^{(0)}, x^{(1)}, \ldots, x^{(n)}, \ldots\right), \quad x^{(n)}=\left(x_{k}^{(n)}\right) \in \ell_{1}
$$

and

$$
\|x\|=\|x\|_{\ell_{1, \infty}^{\omega}}=\max \left(\sum_{n=1}^{\infty} \omega^{n}\left\|x^{(n)}\right\|_{\ell_{1}} \sup _{n, k}\left|x_{k}^{(n)}\right|\right) .
$$

We denote by $\Lambda_{1}^{\omega}$ the direct sum of two copies of $\ell_{1, \infty}^{\omega}, \Lambda_{1}^{\omega}=\ell_{1, \infty}^{\omega} \oplus \ell_{1, \infty}^{\omega}$. Elements of $\Lambda_{1}^{\omega}$ will be denoted by $(y \mid x), y \in \ell_{1, \infty}^{\omega}, x \in \ell_{1, \infty}^{\omega}$ and $\|(y \mid x)\|=\|y\|_{\ell_{1, \infty}^{\omega}}+\|x\|_{\ell_{1, \infty}^{\omega}}^{\omega}$. In other words, 
any element $z \in \Lambda^{\omega}$ can be represented as

$$
z=(y \mid x)=\left(\begin{array}{c:c}
\ldots y_{k}^{(0)} \ldots y_{1}^{(0)} & x_{1}^{(0)} \ldots x_{k}^{(0)} \ldots \\
\ldots & \ldots \\
\ldots y_{k}^{(n)} \ldots y_{1}^{(n)} & x_{1}^{(n)} \ldots x_{k}^{(n)} \ldots \\
\ldots & \ldots
\end{array}\right)
$$

or

$$
z=\sum_{n=0}^{\infty} \sum_{k=1}^{\infty} x_{k}^{(n)} e_{k}^{(n)}+\sum_{n=0}^{\infty} \sum_{k=1}^{\infty} y_{k}^{(n)} e_{k}^{-(n)}
$$

where

$$
x_{k}^{(n)} e_{k}^{(n)}=\left(\begin{array}{c|c}
\ldots 0 \ldots 0 & 0 \ldots 0 \ldots \\
\ldots & \ldots \\
\ldots 0 \ldots 0 & 0 \ldots 0 x_{k}^{(n)} 0 \ldots \\
\ldots 0 \ldots 0 & 0 \ldots 0 \ldots \\
\ldots & \ldots
\end{array}\right)
$$

and

$$
y_{k}^{(n)} e_{k}^{-(n)}=\left(\begin{array}{c|c}
\ldots 0 \ldots 0 & 0 \ldots 0 \ldots \\
\ldots & \ldots \\
\ldots 0 y_{k}^{(n)} 0 \ldots 0 & 0 \ldots 0 \ldots \\
\ldots 0 \ldots 0 & 0 \ldots 0 \ldots \\
\ldots & \ldots
\end{array}\right) .
$$

Note that the expansion (2) is formal, that is, the series on the right is not convergent in general.

We denote by $\Lambda_{1}^{\omega+}$ and $\Lambda_{1}^{\omega-}$ subspaces $\left\{(0 \mid x): x \in \ell_{1, \infty}^{\omega}\right\}$ and $\left\{(y \mid 0): y \in \ell_{1, \infty}^{\omega}\right\}$ respectively. If $z=(y \mid x)$ we will use also notations $z_{+}=x$ and $z_{-}=y$ when it will be convenient.

Let us define the following polynomials on $\Lambda_{1}^{\omega}$

$$
\begin{aligned}
T_{m}^{\omega}(y \mid x) & =\sum_{n=0}^{\infty} \omega^{n} F_{m}^{(n)}\left(x^{(n)}\right)-\sum_{n=0}^{\infty} \omega^{n} F_{m}^{(n)}\left(y^{(n)}\right) \\
& =\sum_{n=0}^{\infty} \omega^{n} \sum_{k=1}^{\infty}\left(x_{k}^{(n)}\right)^{m}-\sum_{n=0}^{\infty} \omega^{n} \sum_{k=1}^{\infty}\left(y_{k}^{(n)}\right)^{m}, \quad(y \mid x) \in \Lambda_{1}^{\omega} .
\end{aligned}
$$

Proposition 1. For every $m \in \mathbb{N}$ the polynomial $T_{m}^{\omega}$ is continuous on $\Lambda_{1}^{\omega}$ and $\left\|T_{m}\right\|=1$.

Proof. Let $\|(y \mid x)\| \leq 1$. Then $\|y\|_{\ell_{1}^{\omega}}+\|x\|_{\ell_{1}^{\omega}} \leq 1$, and $\left|x_{k}^{(n)}\right| \leq 1$ and $\left|y_{k}^{(n)}\right| \leq 1$ for all $k \in \mathbb{N}$ and $n \in \mathbb{Z}_{+}$. Thus

$$
\left|T_{m}^{\omega}(x)\right| \leq \sum_{n=0}^{\infty} \omega^{n} \sum_{k=1}^{\infty}\left(\left|x_{k}^{(n)}\right|^{m}+\left|y_{k}^{(n)}\right|^{m}\right) \leq \sum_{n=0}^{\infty} \omega^{n} \sum_{k=1}^{\infty}\left(\left|x_{k}^{(n)}\right|+\left|y_{k}^{(n)}\right|\right) \leq\|(y \mid x)\| .
$$

So $\left\|T_{m}\right\| \leq 1$. Let now $(y \mid x)$ be such that $y=0, x^{(0)}=(1,0,0, \ldots), x^{(n)}=0$ for $n>0$. Then $\|(y \mid x)\|=1$ and $T_{m}(y \mid x)=1$. Thus $\left\|T_{m}\right\|=1$.

Definition 1. Let us say that a polynomial $P: \Lambda_{1}^{\omega} \rightarrow \mathbb{C}$ is $\omega$-supersymmetric if it is an algebraic combination of polynomials $T_{m}^{\omega}, m \in \mathbb{N}$. We denote by $\mathcal{P}_{s}^{\omega}=\mathcal{P}_{s}^{\omega}\left(\Lambda_{1}^{\omega}\right)$ the algebra of all $\omega$ supersymmetric polynomials on $\Lambda_{1}^{\omega}$. 
Theorem 1. Let $\omega=1 / N$ for some $N \in \mathbb{N}, N>1$. For every number $a \in \mathbb{R}$ there exists $z_{\{a\}} \in \Lambda_{1}^{\omega}$ such that

$$
\left\|z_{\{a\}}\right\|=\left\{\begin{array}{cc}
|a| & \text { if }|a| \geq 1 \\
1 & \text { if }|a|<1
\end{array}\right.
$$

and $T_{m}^{\omega}\left(z_{\{a\}}\right)=a$ for every $m \in \mathbb{N}$.

Proof. Let $a>0$. Then we can write

$$
a=\sum_{j=0}^{\infty} \frac{a_{j}}{N^{j}}, \quad a_{j} \in \mathbb{N},
$$

that is, $a_{0}=[a]$ the integer part of $a$ and $\left(0 . a_{1} a_{2} \ldots\right)_{N}$ is the representation of $a-[a]$ in the positional base $N$ numeral system. Let $z_{\{a\}}$ be of the form $z_{\{a\}}=\left(0 \mid x_{\{a\}}\right)$, where

$$
x_{\{a\}}=\sum_{n=0}^{\infty} x_{\{a\}}^{(n)}
$$

and

$$
x_{\{a\}}^{(n)}=(\underbrace{1, \ldots, 1}_{a_{n}}, 0,0, \ldots)=e_{1}^{(n)}+e_{2}^{(n)}+\cdots+e_{a_{n}}^{(n)}, \quad n=0,1,2, \ldots .
$$

Then for $|a| \geq 1$,

$$
\left\|z_{\{a\}}\right\|=\max \left(\sum_{n=0}^{\infty} \frac{a_{n}}{N^{n}}, 1\right)=\sum_{n=0}^{\infty} \frac{a_{n}}{N^{n}}=T_{m}^{\omega}\left(z_{\{a\}}\right)=a, \quad m \in \mathbb{N}
$$

and $\left\|z_{\{a\}}\right\|=1$ for $|a|<1$. If $a<0$ we can consider $b=-a>0$. By the same way, using (4) for $b$, we can find the vector $x_{\{b\}}$. Let us define now $z_{\{a\}}=\left(x_{\{b\}} \mid 0\right)$. Then

$$
\left\|z_{\{a\}}\right\|=\left\{\begin{array}{cc}
\mu=|a| & \text { if }|a| \geq 1, \\
1 & \text { if }|a|<1,
\end{array}\right.
$$

and $T_{m}^{\omega}\left(z_{\{a\}}\right)=a$ for every $m \in \mathbb{N}$.

Let us recall that two operations on $\ell_{1}$ "•" and " $\diamond$ " which preserve symmetric polynomials were introduced in [7] and [5]. Namely, let $x=\left(x_{1}, x_{2}, \ldots, x_{k}, \ldots\right)$ and $x=\left(y_{1}, y_{2}, \ldots, y_{k}, \ldots\right)$ are in $\ell_{1}$, then

$$
x \bullet y=\left(x_{1}, y_{1}, x_{2}, y_{2}, \ldots, x_{k}, y_{k}, \ldots\right)
$$

and $x \diamond y$ is the resulting sequence of ordering the set $\left\{x_{i} y_{j}: i, j \in \mathbb{N}\right\}$ with one single index in some fixed order. It is easy to check that for every symmetric polynomial $P$ on $\ell_{1}$ and fixed $y \in \ell_{1}$, polynomials $P(x \bullet y)$ and $P(x \diamond y)$ are symmetric. In [11] these operations were extended to $\ell_{1} \oplus \ell_{1}$ with preserving supersymmetric polynomials. Now we propose natural extensions of these operations to $\Lambda_{1}^{\omega}$.

Definition 2. Let $z=\left(z_{-} \mid z_{+}\right)$and $r=\left(r_{-} \mid r_{+}\right)$are in $\Lambda_{1}^{\omega}$. We say that $h=z \bullet r$ if $h_{-}^{(n)}=$ $z_{-}^{(n)} \bullet r_{-}^{(n)}$ and $h_{+}^{(n)}=z_{+}^{(n)} \bullet r_{+}^{(n)}$ for every $n \in \mathbb{Z}_{+}$. We also say that $s=z \diamond r$ if

$s_{+}^{(n)}=\left(z_{+}^{(0)} \diamond r_{+}^{(n)}\right) \bullet\left(z_{+}^{(1)} \diamond r_{+}^{(n-1)}\right) \bullet \cdots \bullet\left(z_{+}^{(n)} \diamond r_{+}^{(0)}\right) \bullet\left(z_{-}^{(0)} \diamond r_{-}^{(n)}\right) \bullet\left(z_{-}^{(1)} \diamond r_{-}^{(n-1)}\right) \bullet \cdots \bullet\left(z_{-}^{(n)} \diamond r_{-}^{(0)}\right)$

and

$s_{-}^{(n)}=\left(z_{+}^{(0)} \diamond r_{-}^{(n)}\right) \bullet\left(z_{+}^{(1)} \diamond r_{-}^{(n-1)}\right) \bullet \cdots \bullet\left(z_{+}^{(n)} \diamond r_{-}^{(0)}\right) \bullet\left(z_{-}^{(0)} \diamond r_{+}^{(n)}\right) \bullet\left(z_{-}^{(1)} \diamond r_{+}^{(n-1)}\right) \bullet \cdots \bullet\left(z_{-}^{(n)} \diamond r_{+}^{(0)}\right)$. 
Proposition 2. $T_{m}^{\omega}(z \bullet r)=T_{m}^{\omega}(z)+T_{m}^{\omega}(r)$ and $T_{m}^{\omega}(z \diamond r)=T_{m}^{\omega}(z) T_{m}^{\omega}(r)$ for all $z, r \in \Lambda_{1}^{\omega}$ and $m \in \mathbb{N}$.

Proof. The first equality directly follows from the definition of $T_{m}^{\omega}$ (3). Also, in [5] it is proved that $F_{m}(x \diamond y)=F_{m}(x) F_{m}(y), x, y \in \ell_{1}, m \in \mathbb{N}$. So, using (3) and Definition 2, we have for $s=z \diamond r$

$$
\begin{aligned}
T_{m}^{\omega}(s)= & T_{m}^{\omega}(z \diamond r)=\sum_{n=0}^{\infty} \omega^{n} F_{m}^{(n)}\left(s_{+}^{(n)}\right)-\sum_{n=0}^{\infty} \omega^{n} F_{m}^{(n)}\left(s_{-}^{(n)}\right) \\
= & \sum_{n=0}^{\infty} \omega^{n}\left(\sum_{j=0}^{n} F_{m}^{(n)}\left(z_{+}^{(j)} \diamond r_{+}^{(n-j)}\right)+\sum_{j=0}^{n} F_{m}^{(n)}\left(z_{-}^{(j)} \diamond r_{-}^{(n-j)}\right)\right) \\
& \quad-\sum_{n=0}^{\infty} \omega^{n}\left(\sum_{j=0}^{n} F_{m}^{(n)}\left(z_{+}^{(j)} \diamond r_{-}^{(n-j)}\right)+\sum_{j=0}^{n} F_{m}^{(n)}\left(z_{-}^{(j)} \diamond r_{+}^{(n-j)}\right)\right) \\
= & \sum_{n=0}^{\infty} \omega^{n}\left(\sum_{j=0}^{n} F_{m}^{(j)}\left(z_{+}^{(j)}\right) F_{m}^{(n-j)}\left(r_{+}^{(n-j)}\right)+\sum_{j=0}^{n} F_{m}^{(j)}\left(z_{-}^{(j)}\right) F_{m}^{(n-j)}\left(r_{-}^{(n-j)}\right)\right) \\
& -\sum_{n=0}^{\infty} \omega^{n}\left(\sum_{j=0}^{n} F_{m}^{(j)}\left(z_{+}^{(j)}\right) F_{m}^{(n-j)}\left(r_{-}^{(n-j)}\right)+\sum_{j=0}^{n} F_{m}^{(j)}\left(z_{-}^{(j)}\right) F_{m}^{(n-j)}\left(r_{+}^{(n-j)}\right)\right) \\
= & \left(\sum_{n=0}^{\infty} \omega^{n} F_{m}^{(n)}\left(z_{+}^{(n)}\right)-\sum_{n=0}^{\infty} \omega^{n} F_{m}^{(n)}\left(z_{-}^{(n)}\right)\right)\left(\sum_{n=0}^{\infty} \omega^{n} F_{m}^{(n)}\left(r_{+}^{(n)}\right)-\sum_{n=0}^{\infty} \omega^{n} F_{m}^{(n)}\left(r_{-}^{(n)}\right)\right) \\
= & T_{m}^{\omega}(z) T_{m}^{\omega}(r) .
\end{aligned}
$$

Corollary 1. Let $P(z) \in \mathcal{P}_{s}^{\omega}$. Then, for every fixed $r \in \Lambda_{1}^{\omega}$ polynomials $P(z \bullet r)$ and $P(z \diamond r)$ are in $\mathcal{P}_{s}^{\omega}$.

For a given $z=(y \mid x) \in \Lambda_{1}^{\omega}$ we denote $z^{-}=(x \mid y)$. Clearly, the map $z \mapsto z^{-}$is a continuous involution in $r \in \Lambda_{1}^{\omega}$ and $T_{m}^{\omega}\left(z^{-}\right)=-T_{m}^{\omega}(z)$.

Let us introduce the following relation of equivalence on $\Lambda_{1}^{\omega}$. We say that $z \sim r$ if and only if $T_{m}^{\omega}(z)=T_{m}^{\omega}(r)$ for every $m \in \mathbb{N}$. Let us denote by $\mathcal{M}^{\omega}$ the quotient set $\Lambda_{1}^{\omega} / \sim$ and by $[z]$ the class of equivalence which contains $z$.

Proposition 3. The following operations $[z]+[r]:=[z \bullet r] ;[z][r]:=[z \diamond r], z, r \in \Lambda_{1}^{\omega}$, of addition and multiplication are well-defined on $\mathcal{M}^{\omega} \times \mathcal{M}^{\omega}$ and $\left(\mathcal{M}^{\omega},+, \cdot\right)$ is a unital commutative ring.

Proof. Let $z^{\prime} \in[z]$ and $r^{\prime} \in[r]$. By Proposition 2 and the definition of the equivalence we have that for every $m \in \mathbb{N}$,

$$
T_{m}^{\omega}(z)+T_{m}^{\omega}(r)=T_{m}^{\omega}\left(z^{\prime}\right)+T_{m}^{\omega}\left(r^{\prime}\right)=T_{m}^{\omega}\left(z^{\prime} \bullet r^{\prime}\right)
$$

and

$$
T_{m}^{\omega}(z) T_{m}^{\omega}(r)=T_{m}^{\omega}\left(z^{\prime}\right) T_{m}^{\omega}\left(r^{\prime}\right)=T_{m}^{\omega}\left(z^{\prime} \diamond r^{\prime}\right)
$$

So the operations on $\mathcal{M}^{\omega}$ do not depend on representatives. Let $[u]=[z]([r]+[s])$ and $[v]=$ $[z][r]+[z][s]$. Since for every $m \in \mathbb{N}$

$$
T_{m}^{\omega}(u)=T_{m}^{\omega}(z)\left(T_{m}^{\omega}(r)+T_{m}^{\omega}(s)\right)=T_{m}^{\omega}(z) T_{m}^{\omega}(r)+T_{m}^{\omega}(z) T_{m}^{\omega}(s)=T_{m}^{\omega}(v),
$$


so $[u]=[v]$ and we have the distributive law. Clearly that the associativity and commutativity of the addition and multiplication can be proved by the same way. Also, $-[z]=\left[z^{-}\right]$and $\mathbb{I}=\left[e_{1}^{(0)}\right]$ is the identity. Thus $\mathcal{M}^{\omega}$ is a unital commutative ring.

For any $\lambda \in \mathbb{C}$ and $z \in \mathcal{M}^{\omega}$ we set $\lambda *[z]=[\lambda z]$. Since, $T_{m}^{\omega}(\lambda z)=\lambda^{m} T_{m}^{\omega}(z)$, the operation " $*$ " is well defined on $\mathbb{C} \times \mathcal{M}^{\omega}$. But $\left(\mathcal{M}^{\omega},+, *\right)$ is not a linear space. Indeed, if $z \in \Lambda_{1}^{\omega}$ and $z \neq 0$, then $[z]+[z]=[z \bullet z] \neq 2 *[z]$ because $T_{m}^{\omega}([z \bullet z])=2 T_{m}^{\omega}(z)$ but $T_{m}^{\omega}(2 z)=2^{m} T_{m}^{\omega}(z)$.

\section{OpERATORS AND SEMINORMS ON $\mathcal{M}^{1 / N}$}

For a given $z=(y \mid x) \in \Lambda_{1}^{\omega}$, we denote by $\operatorname{supp} z$ the support of $z$, that is, the following pair of sets of indexes

$$
\operatorname{supp} z=\left(\left\{i \in \mathbb{N}, j \in \mathbb{Z}_{+}: y_{i}^{(j)} \neq 0\right\},\left\{k \in \mathbb{N}, n \in \mathbb{Z}_{+}: x_{k}^{(n)} \neq 0\right\}\right) .
$$

Let us define the following maps on $\Lambda_{1}^{1 / N}$ :

$$
S_{k}^{+(n, m)}(z)=\left(z-x_{k}^{(n)} e_{k}^{(n)}\right) \bullet(\underbrace{x_{k}^{(m)} e_{k}^{(m)} \bullet \cdots \bullet\left(x_{k}^{(m)} e_{k}^{(m)}\right.}_{N^{m-n}})
$$

and

$$
S_{k}^{-(n, m)}(z)=\left(z-y_{k}^{(n)} e_{k}^{-(n)}\right) \bullet(\underbrace{y_{k}^{(m)} e_{k}^{-(m)} \bullet \cdots \bullet\left(y_{k}^{(m)} e_{k}^{-(m)}\right.}_{N^{m-n}}),
$$

where $m \geq n$ and $z=(y \mid x) \in \Lambda_{1}^{1 / N}$ for some $N \in \mathbb{N}, N>1$. Let $\sigma: \mathbb{N} \rightarrow \mathbb{N}$ be a permutation. We denote by $S_{\sigma}^{+(i)}$ and $S_{\sigma}^{-(i)}$ linear operators on $\Lambda_{1}^{1 / N}$ such that

$$
S_{\sigma}^{+(i)}\left(e_{k}^{(j)}\right)=e_{\sigma(k)}^{(i)} \text { if } i=j \text { and } S_{\sigma}^{+(i)}\left(e_{k}^{ \pm(j)}\right)=e_{k}^{ \pm(j)} \text { otherwise, }
$$

and

$$
S_{\sigma}^{-(i)}\left(e_{k}^{-(i)}\right)=e_{\sigma(k)}^{-(i)} \text { if } i=j \text { and } S_{\sigma}^{-(i)}\left(e_{k}^{ \pm(j)}\right)=e_{k}^{ \pm(j)} \text { otherwise. }
$$

Lemma 1. For every $z=(y \mid x) \in \Lambda_{1}^{1 / N}$, permutation $\sigma$ on $\mathbb{N}$ and $m \geq n$ we have

$$
[z]=\left[S_{\sigma}^{+(i)}(z)\right]=\left[S_{\sigma}^{-(i)}(z)\right]=\left[S_{k}^{+(n, m)}(z)\right]=\left[S_{k}^{-(n, m)}(z)\right] .
$$

Proof. The proof follows from the definitions and direct calculations.

Proposition 4. Let $z=(y \mid x) \in \Lambda_{1}^{1 / N}$ for some $N \in \mathbb{N}, N>1$ and $z$ has a finite support. If $[z]=[0]$, then there is a number $j \in \mathbb{N}$ and a composition $S$ of a finite set of mappings $\left\{S_{k}^{ \pm(n, m)}, S_{\sigma}^{ \pm(j)}\right\}$ defined above such that

$$
S(z)=\left(y^{\prime} \mid x^{\prime}\right)=\left(\begin{array}{c|c}
\ldots 0 \ldots 0 & 0 \ldots 0 \ldots \\
\ldots & \ldots \\
\ldots 0 \ldots 0 & 0 \ldots 0 \ldots \\
\ldots y_{k}^{\prime(j)} \ldots y_{1}^{\prime(j)} & x_{1}^{\prime(j)} \ldots x_{k}^{(j)} \ldots \\
\ldots 0 \ldots 0 & 0 \ldots 0 \ldots \\
\ldots & \ldots
\end{array}\right)=\sum_{k=1}^{\infty} x_{k}^{\prime(j)} e_{k}^{(j)}+\sum_{k=1}^{\infty} y_{k}^{\prime(j)} e_{k}^{-(j)}
$$

and $x_{k}^{\prime(j)}=y_{k}^{\prime(j)}$ for every $k \in \mathbb{N}$. 
Proof. Let $j$ be a minimal number such that $x_{k}^{(j)}=0$ and $y_{k}^{(j)}$ for every $k \in \mathbb{N}$. Using a finite number of mappings $S_{k}^{ \pm(n, m)}$ and Lemma 1 we can find $z^{\prime}=\left(y^{\prime} \mid x^{\prime}\right), z^{\prime} \sim z$ which satisfies (5). So, for every $m \in \mathbb{N}$

$$
\sum_{k=1}^{\infty}\left(y_{k}^{\prime(j)}\right)^{m}=\sum_{k=1}^{\infty}\left(x_{k}^{\prime(j)}\right)^{m} .
$$

From [1] it follows that vectors $\left(y_{k}^{\prime(j)}\right)_{k}$ and $\left(x_{k}^{\prime(j)}\right)_{k}$ coincide up to a permutation $\sigma$ of coordinates $\left(x_{1}, \ldots, x_{k}, \ldots\right)$. So, applying $S_{\sigma}^{(j)}$ to $z^{\prime}$ we have $x_{k}^{\prime(j)}=y_{k}^{\prime(j)}$ for every $k \in \mathbb{N}$.

Corollary 2. Let $z=(y \mid x) \in \Lambda_{1}^{1 / N}$ for some $N \in \mathbb{N}, N>1$, and $z$ has a finite support. Then there is an element $z^{\prime}=\left(y^{\prime} \mid x^{\prime}\right) \in \Lambda_{1}^{1 / N}$ such that $z \sim z^{\prime}$ and $z^{\prime}$ has the following property: if ${y^{\prime}}_{i}^{(j)} \neq 0$, then $x_{k}^{\prime(n)} \neq y_{i}^{\prime(j)}$ for all $k \in \mathbb{N}, n \in \mathbb{Z}_{+}$.

Proof. To get a proof it is enough to apply Proposition 4 to $z \bullet z^{\prime-}=\left(y \bullet x^{\prime} \mid x \bullet y^{\prime}\right)$.

Due to Theorem 1, we can introduce an alternative multiplication by real constants in $\mathcal{M}^{\omega}$, at least for the case $\omega=1 / N, N \in \mathbb{N}, N>1$.

Theorem 2. Let $N \in \mathbb{N}, N>1$. Then $\mathcal{M}^{1 / N}$ is a real linear commutative unital algebra with respect to the operations of addition and multiplication defined in Proposition 3 and the following multiplication by constants:

$$
a[z]:=\left[z_{\{a\}}\right][z]=\left[z_{\{a\}} \diamond z\right], \quad a \in \mathbb{R},
$$

where $z_{\{a\}}$ is as in Theorem 1.

Proof. Note first that from Theorem 1 and Proposition 2 it follows that for every $m \in \mathbb{N}$, $T_{m}^{\omega}\left(z_{\{a\}} \diamond z\right)=a T_{m}^{\omega}(z)$. So $\mathbb{I}=z_{\{1\}}$ is the unity in $\mathcal{M}^{1 / N}$ and $\left[z_{\left\{a_{1}+a_{2}\right\}}\right]=\left[z_{\left\{a_{1}\right\}}\right]+\left[z_{\left\{a_{2}\right\}}\right]$, $a_{1}, a_{2} \in \mathbb{R}$. Thus,

$$
a([z]+[r])=a[z]+a[r] \quad \text { and } \quad\left(a_{1}+a_{2}\right)[z]=a_{1}[z]+a_{2}[z]
$$

where $a, a_{1}, a_{2} \in \mathbb{R}$ and $[z],[r] \in \mathcal{M}^{1 / N}$.

Let us denote by $\Omega$ the class of functions $\gamma: \mathbb{C} \rightarrow \mathbb{C}$ such that the mappings $\Phi_{\gamma}: \Lambda_{1}^{\omega} \rightarrow \Lambda_{1}^{\omega}$ defined by

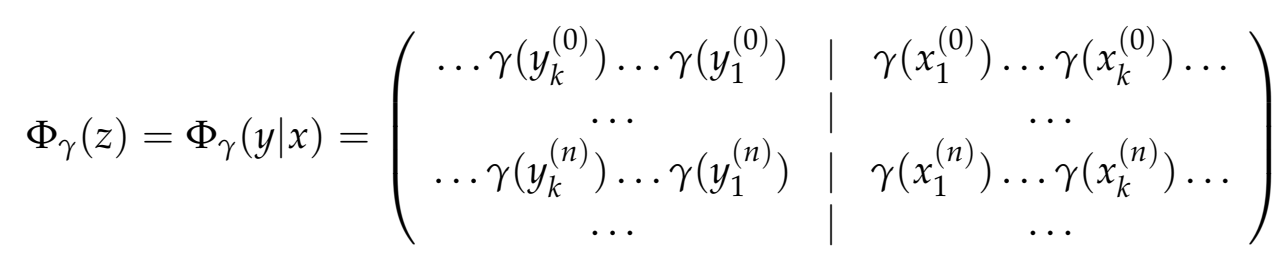

are well defined and $z \sim z^{\prime}$ implies $\Phi_{\gamma}(z)=\Phi_{\gamma}\left(z^{\prime}\right)$. Such class is nonempty, for example, $\gamma(t)=t^{m} \in \Omega, m \in \mathbb{N}$.

Theorem 3. Let $\gamma \in \Omega$. Then $\Phi_{\gamma}$ generates a linear operator $\widehat{\Phi}_{\gamma}: \mathcal{M}^{1 / N} \rightarrow \mathcal{M}^{1 / N}$ defined by $\widehat{\Phi}_{\gamma}([z])=\Phi_{\gamma}(z)$. 
Proof. From the definition of $\Omega$ it follows that $\widehat{\Phi}_{\gamma}$ is well defined. Also, it is clear

$$
\widehat{\Phi}_{\gamma}([z]+[r])=\Phi_{\gamma}(z \bullet r)=\Phi_{\gamma}(z) \bullet \Phi_{\gamma}(r)=\widehat{\Phi}_{\gamma}([z])+\widehat{\Phi}_{\gamma}([r]),
$$

$z, r \in \Lambda_{1}^{1 / N}$. Let now $z_{\{a\}}=\left(y_{\{a\}} \mid x_{\{a\}}\right)$ be as in Theorem 1, that is,

$$
x_{\{a\}}=\sum_{n=0}^{\infty} \sum_{i=1}^{a_{n}} e_{i}^{(n)}, \quad y_{\{a\}}=0 \text { if } a \geq 0 \quad \text { and } \quad y_{\{a\}}=\sum_{n=0}^{\infty} \sum_{i=1}^{a_{n}} e_{i}^{-(n)}, \quad x_{\{a\}}=0 \text { if } a<0,
$$

where

$$
|a|=\sum_{j=0}^{\infty} \frac{a_{j}}{N^{j}}, \quad a_{j} \in \mathbb{N} .
$$

If $a \geq 0$, then $\left[z_{\{a\}}\right][z]=a[z], a \in \mathbb{R}, z=(y \mid x) \in \Lambda_{1}^{1 / N}$ and

$$
\begin{gathered}
\Phi_{\gamma}\left(z_{\{a\}} \diamond z\right)=\Phi_{\gamma}\left((\underbrace{z \bullet \ldots \bullet z}_{a_{0}}) \diamond e_{1}^{(0)} \bullet \ldots \bullet(\underbrace{z \bullet \ldots \bullet z}_{a_{n}}) \diamond e_{1}^{(n)} \bullet \ldots\right) \\
=(\underbrace{\Phi_{\gamma}(z) \bullet \ldots \bullet \Phi_{\gamma}(z)}_{a_{0}}) \diamond e_{1}^{(0)} \bullet \ldots \bullet(\underbrace{\Phi_{\gamma}(z) \bullet \ldots \bullet \Phi_{\gamma}(z)}_{a_{n}}) \diamond e_{1}^{(n)} \bullet \ldots=z_{\{a\}} \diamond \Phi_{\gamma}(z) .
\end{gathered}
$$

If $a<0$, we have to replace $e_{1}^{(n)}$ by $e_{1}^{-(n)}, n \in \mathbb{Z}_{+}$. So $\widehat{\Phi}_{\gamma}(a[z])=a \widehat{\Phi}_{\gamma}([z])$. Therefore, $\widehat{\Phi}_{\gamma}$ is a linear operator.

Let us denote $\tau_{m}([z])=T_{m}^{1 / N}(z),[z] \in \mathcal{M}^{1 / N}, m \in \mathbb{N}$. Clearly, $\tau_{m}$ are complex valued real-linear and multiplicative functions, that is, $\tau_{m}$ are homomorphisms from $\mathcal{M}^{1 / N}$ to $\mathbb{C}$. By the definition of $\mathcal{M}^{1 / N}$ we have that functionals $\tau_{m}: m \in \mathbb{N}$ separate points of $\mathcal{M}^{1 / N}$. Let us denote by $\bar{z}=\Phi_{\gamma}(z)$, where $\gamma(t)=\bar{t}$ is the complex conjugate of $t$. It is easy to check that $\tau_{m}([\bar{z}])=\overline{\tau_{m}([z])}$ and so $\gamma(t)=\bar{t}$ belongs to $\Omega$. So $[z] \mapsto \tau_{m}([\bar{z}])$ is a complex valued functional for every $m \in \mathbb{N}$. Thus $\tau_{m}+\bar{\tau}_{m}$ and $-i\left(\tau_{m}-\bar{\tau}_{m}\right)$ are real valued linear functionals on $\mathcal{M}^{1 / N}$.

Corollary 3. If $\gamma \in \Omega$ is multiplicative, then $\widehat{\Phi}_{\gamma}$ is an algebra homomorphism.

Proof. Let $[z],[r] \in \mathcal{M}^{1 / N}$,

$$
z=\sum_{n=0}^{\infty} \sum_{k=1}^{\infty} z_{+k}^{(n)} e_{k}^{(n)}+\sum_{n=0}^{\infty} \sum_{k=1}^{\infty} z_{-k}^{(n)} e_{k}^{-(n)}
$$

and

$$
r=\sum_{n=0}^{\infty} \sum_{k=1}^{\infty} r_{+k}^{(n)} e_{k}^{(n)}+\sum_{n=0}^{\infty} \sum_{k=1}^{\infty} r_{-k}^{(n)} e_{k}^{-(n)}
$$

Since $\Phi_{\gamma}\left(z_{+k}^{(n)} e_{k}^{(n)}\right)=\gamma\left(z_{+k}^{(n)}\right) e_{k}^{(n)}$, we have

$$
\Phi_{\gamma}\left(z_{ \pm k}^{(n)} e_{k}^{ \pm(n)} \diamond r_{ \pm i}^{(j)} e_{i}^{ \pm(j)}\right)=\gamma\left(z_{ \pm k}^{(n)} r_{ \pm i}^{(j)}\right) e_{k}^{ \pm(n)} \diamond e_{i}^{ \pm(j)},
$$

$k, i \in \mathbb{N}, n, j \in \mathbb{Z}_{+}$. From the linearity and multiplicativity of $\tau_{m}$ it follows

$$
\tau_{m}\left(\widehat{\Phi}_{\gamma}([z])\right) \tau_{m}\left(\widehat{\Phi}_{\gamma}([r])\right)=\tau_{m}\left(\widehat{\Phi}_{\gamma}([z]) \widehat{\Phi}_{\gamma}([r])\right)=\tau_{m}\left(\widehat{\Phi}_{\gamma}([z][r])\right) .
$$

Since it is true for every $m$, we have

$$
\widehat{\Phi}_{\gamma}([z]) \widehat{\Phi}_{\gamma}([r])=\widehat{\Phi}_{\gamma}([z][r]) .
$$


Proposition 5. Let $\gamma \in \Omega$ and $\gamma(0)=0$. Then the following formula defines a seminorm on $\mathcal{M}^{1 / N}$ :

$$
p_{\gamma}([z])=\inf _{(y \mid x) \in[z]} \sum_{n=0}^{\infty} \frac{1}{N^{n}} \sum_{k=1}^{\infty}\left(\left|\gamma\left(x_{k}^{(n)}\right)\right|+\left|\gamma\left(y_{k}^{(n)}\right)\right|\right) .
$$

Proof. Since the infimum is taken over all representations $(y \mid x) \in[z]$, the norm is well defined. It is easy to check that $p_{\gamma}$ is nonnegative and satisfies the triangle inequality and is homogeneous.

Definition 3. Let us define the following seminorms on $\mathcal{M}^{1 / N}$ :

$$
p_{m}([z])=p_{\gamma_{m}}([z]) \text { for } \gamma_{n}(t)=t^{m} .
$$

It is clear that $\left|\tau_{m}([z])\right| \leq p_{m}([z]),[z] \in \mathcal{M}^{1 / N}$ and so, if $[z] \neq 0$, then there is $m \in \mathbb{N}$ such that $p_{m}([z])>0$.

Let us denote $\left(\mathcal{M}^{1 / N},\left(p_{m}\right)\right)$ the linear space $\mathcal{M}^{1 / N}$ endowed with the projective topology, generated by seminorms $\left(p_{m}\right)$. So we have the following proposition.

Proposition 6. The space $\left(\mathcal{M}^{1 / N},\left(p_{m}\right)\right)$ is a locally convex metrisable topological vector space and each functional $\tau_{m}$ is continuous on $\left(\mathcal{M}^{1 / N},\left(p_{m}\right)\right)$.

Let us denote by $\mathcal{D}$ the following subset of $\mathcal{M}^{1 / N}$ :

$$
\mathcal{D}=\left\{u \in \mathcal{M}^{1 / N}: \text { there is } z \in u \text { such that }\left|z_{k}^{(n)}\right| \leq 1, n \in \mathbb{Z}_{+}, k \in \mathbb{N}\right\} .
$$

Theorem 4. $\mathcal{D}$ is a subalgebra in $\mathcal{M}^{1 / N}$ and the restriction of the topology of $\left(\mathcal{M}^{1 / N},\left(p_{n}\right)\right)$ to $\mathcal{D}$ is generated by a norm on $\mathcal{D}$.

Proof. From the definition of addition and multiplication in $\mathcal{M}^{1 / N}$ it follows that $u+v \in \mathcal{D}$ and $u v \in \mathcal{D}$ for all $u, v \in \mathcal{D}$. Also, for every $a \in \mathbb{R},\left[z_{\{a\}}\right] \in \mathcal{D}$ and so $a u=\left[z_{\{a\}}\right] u \in \mathcal{D}$. Hence, $\mathcal{D}$ is a subalgebra in $\mathcal{M}^{1 / N}$. Note that for every $u \in \mathcal{D}$ and $m \in \mathbb{N}, p_{m}(u) \leq p_{1}(u)$. Also, $p_{1}$ is a norm on $\mathcal{D}$. Indeed, if $u \neq 0$, then there is $m \in \mathbb{N}$ such that $\tau_{m}(u) \neq 0$. So

$$
0 \neq\left|\tau_{m}(u)\right| \leq p_{m}(u) \leq p_{1}(u) .
$$

So $\left(\mathcal{D}, p_{1}\right)$ is a normed space and all $p_{m}$ are continuous with respect to $p_{1}$. So the restriction of topology of $\left(\mathcal{M}^{1 / N},\left(p_{n}\right)\right)$ to $\mathcal{D}$ coincides with the norm topology of $\left(\mathcal{D}, p_{1}\right)$.

\section{REFERENCES}

[1] Alencar R., Aron R., Galindo P., Zagorodnyuk A. Algebra of symmetric holomorphic functions on $\ell_{p}$. Bull. Lond. Math. Soc. 2003, 35, 55-64. doi:10.1112/S0024609302001431

[2] Aron R.M., Cole B.J., Gamelin T.W. Spectra of algebras of analytic functions on a Banach space. J. Reine Angew. Math. 1991, 415, 51-93.

[3] Aron R., Galindo P., Pinasco D., Zalduendo I. Group-symmetric holomorphic functions on a Banach space. Bull. Lond. Math. Soc. 2016, 48 (5), 779-796. doi:10.1112/blms/bdw043

[4] Chernega I.V. A semiring in the spectrum of the algebra of symmetric analytic functions in the space $\ell_{1}$. J. Math. Sci. 2016, 212, 38-45. doi:10.1007/s10958-015-2647-3

[5] Chernega I., Galindo P., Zagorodnyuk A. A multiplicative convolution on the spectra of algebras of symmetric analytic functions. Rev. Mat. Complut. 2014, 27 (2), 575-585. doi:10.1007/s13163-013-0128-0 
[6] Chernega I., Galindo P., Zagorodnyuk A. Some algebras of symmetric analytic functions and their spectra. Proc. Edinb. Math. Soc. 2012, 55, 125-142. doi:10.1017/S0013091509001655

[7] Chernega I., Galindo P., Zagorodnyuk A. The convolution operation on the spectra of algebras of symmetric analytic functions. J. Math. Anal. Appl. 2012, 395 (2), 569-577. doi:10.1016/j.jmaa.2012.04.087

[8] Chernega I., Holubchak O., Novosad Z., Zagorodnyuk A. Continuity and hypercyclicity of composition operators on algebras of symmetric analytic functions on Banach spaces. Eur. J. Math. 2019, 12 P. doi:10.1007/s40879-01900390-z

[9] Dineen S. Complex analysis on infinite-dimensional spaces. Springer-Verlag, London, 1999.

[10] Jawad F. Note on separately symmetric polynomials on the Cartesian product of $\ell_{1}$. Mat. Stud. 2018, 50 (2), $204-210$. doi:10.15330/ms.50.2.204-210

[11] Jawad F., Zagorodnyuk A. Supersymmetric Polynomials on the Space of Absolutely Convergent Series. Symmetry 2019, 11 (9), 1111 (19 p.). doi:10.3390/sym11091111

[12] Galindo P., Vasylyshyn T., Zagorodnyuk A. The algebra of symmetric analytic functions on $L_{\infty}$. Proc. Roy. Soc. Edinburgh Sect. A. 2107, 147 (4), 743-761. doi:10.1017/S0308210516000287

[13] Galindo P., Vasylyshyn T., Zagorodnyuk A. Symmetric and finitely symmetric polynomials on the spaces $\ell_{\infty}$ and $L_{\infty}[0,+\infty)$. Math. Nachr. 2018, 291 (11-12), 1712-1726. doi:10.1002/mana.201700314

[14] García D., Maestre M., Zalduendo I. The spectra of algebras of group-symmetric functions. Proc. Edinb. Math. Soc. 2019, 62 (3), 609-623. doi:10.1017/S0013091518000603

[15] Gonzaléz M., Gonzalo R., Jaramillo J. Symmetric polynomials on rearrangement invariant function spaces. J. London Math. Soc. 1999, 59, 681-697.

[16] Kravtsiv V., Vasylyshyn T., Zagorodnyuk A. On Algebraic Basis of the Algebra of Symmetric Polynomials on $\ell_{p}\left(\mathbf{C}^{\mathbf{n}}\right)$. J. Funct. Spaces 2017, 2017 (7), Article ID 4947925, 8 p. doi:10.1155/2017/4947925

Received 04.08.2019

Ажавад Ф., Карпенко Г., Загороднюк А. Алгебри, породжені спеціальними симетричними поліномами на $\ell_{1}$ // Карпатські матем. публ. - 2019. - Т.11, №2. - С. 335-344.

Нехай $X$ - зважена пряма сума нескінченної кількості копій комплексного простору $\ell_{1} \oplus \ell_{1}$. Ми розглялаємо алгебру, яка складається з поліномів на $X$, котрі $є$ суперсиметричними на кожному доданку $\ell_{1} \bigoplus \ell_{1}$. Функціонали значень в точках на цій алгебрі задають відношення еквівалентності ' ' на X. У роботі досліджено фактор-множину $X / \sim$ і показано, що за деяких умов на цій множині є структура дійсної топологічної алгебри.

Ключові слова і фрази: симетричні і суперсиметричні поліноми на банахових просторах, алгебри аналітичних функцій на банахових просторах, спектри алгебр аналітичних функцій. 